\title{
The Resource Identification Initiative: A cultural shift in
}

\section{publishing [version 1; peer review: 2 approved]}

\author{
Anita Bandrowski1 ${ }^{1}$ Matthew Brush22, Jeffery S. Grethe (D1), Melissa A. Haendel2, \\ David N. Kennedy (D)3, Sean Hill (iD) 4, Patrick R. Hof5, Maryann E. Martone1, \\ Maaike Pols ${ }^{6}$, Serena Tan7, Nicole Washington ${ }^{8}$, Elena Zudilova-Seinstra9 \\ Nicole Vasilevsky (iD2, \\ Resource Identification Initiative Members are listed here: \\ https://www.force11.org/node/4463/members
}

\footnotetext{
${ }^{1}$ Center for Research in Biological Systems, UCSD, la Jolla, CA, 92093, USA

${ }^{2}$ Department of Medical Informatics \& Clinical Epidemiology, OHSU, Portland, Oregon, 97239, USA

${ }^{3}$ Department of Psychiatry, University of Massachusetts Medical School, Worcester, MA, 01605, USA

${ }^{4}$ Karolinska Institutet, Stockholm, 171 77, Sweden

${ }^{5}$ Leon and Norma Hess Center for Science and Medicine, Icahn School of Medicine at Mount Sinai, New York, NY, 10029, USA

${ }^{6}$ Scientific Outreach, Faculty of 1000 Ltd, London, W1T 4LB, UK

7John Wiley and Sons, Hoboken, NJ, 07030, USA

${ }^{8}$ Lawrence Berkeley National Laboratory, Lawrence Berkeley National Laboratory, Berkeley, CA, 94720, USA

${ }^{9}$ Elsevier, Amsterdam, 1043 NX, The Netherlands
}

\section{V1 First published: 29 May 2015, 4:134 \\ https://doi.org/10.12688/f1000research.6555.1}

Latest published: 19 Nov 2015, 4:134

https://doi.org/10.12688/f1000research.6555.2

\begin{abstract}
A central tenet in support of research reproducibility is the ability to uniquely identify research resources, i.e., reagents, tools, and materials that are used to perform experiments. However, current reporting practices for research resources are insufficient to allow humans and algorithms to identify the exact resources that are reported or answer basic questions such as "What other studies used resource X?" To address this issue, the Resource Identification Initiative was launched as a pilot project to improve the reporting standards for research resources in the methods sections of papers and thereby improve identifiability and reproducibility. The pilot engaged over 25 biomedical journal editors from most major publishers, as well as scientists and funding officials. Authors were asked to include Research Resource Identifiers (RRIDs) in their manuscripts prior to publication for three resource types: antibodies, model organisms, and tools (including software and databases). RRIDs represent accession numbers assigned by an authoritative database, e.g., the model organism databases, for each type of resource. To make it easier for authors to obtain RRIDs, resources were aggregated from the appropriate databases and their RRIDs made available in a central web portal (www.scicrunch.org/resources). RRIDs
\end{abstract}

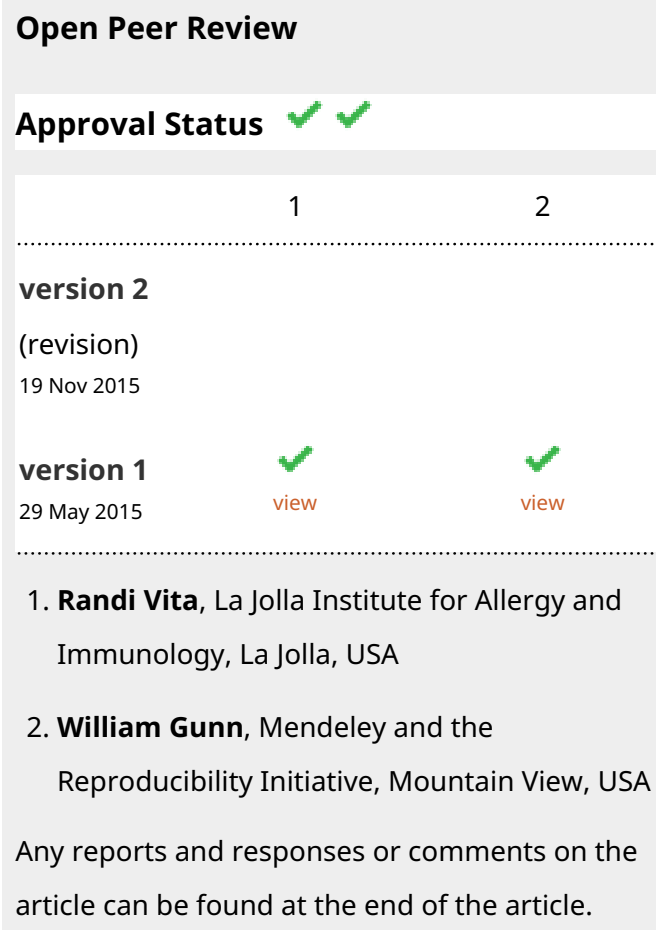

1 2

version 2

(revision)

19 Nov 2015

version 1

29 May 2015

1. Randi Vita, La Jolla Institute for Allergy and Immunology, La Jolla, USA

2. William Gunn, Mendeley and the

Reproducibility Initiative, Mountain View, USA Any reports and responses or comments on the article can be found at the end of the article. 
meet three key criteria: they are machine readable, free to generate and access, and are consistent across publishers and journals. The pilot was launched in February of 2014 and over 300 papers have appeared that report RRIDs. The number of journals participating has expanded from the original 25 to more than 40 . Here, we present an overview of the pilot project and its outcomes to date. We show that authors are generally accurate in performing the task of identifying resources and supportive of the goals of the project. We also show that identifiability of the resources pre- and post-pilot showed a dramatic improvement for all three resource types, suggesting that the project has had a significant impact on reproducibility relating to research resources.

Keywords

Resource identifiers, Multi-centre initiative, Publishing , Pre-pilot data, Post-pilot data

This article is included in the Research on

Research, Policy \& Culture gateway.

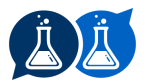

This article is included in the Preclinical

Reproducibility and Robustness gateway.

\section{«incf}

This article is included in the INCF gateway.

Corresponding author: Anita Bandrowski (abandrowski@ncmir.ucsd.edu)

Competing interests: The authors declared no competing interests.

Grant information: This work was supported by: an NIF grant to Martone PI (HHSN271200577531C/PHS HHS/United States); a NIDDK grant to Martone PI (1U24DK097771-01); and a grant from Monarch to Haendel PI (5R24OD011883).

The funders had no role in study design, data collection and analysis, decision to publish, or preparation of the manuscript.

Copyright: (c) 2015 Bandrowski A et al. This is an open access article distributed under the terms of the Creative Commons Attribution License, which permits unrestricted use, distribution, and reproduction in any medium, provided the original work is properly cited.

How to cite this article: Bandrowski A, Brush M, Grethe JS et al. The Resource Identification Initiative: A cultural shift in publishing [version 1; peer review: 2 approved] F1000Research 2015, 4:134 https://doi.org/10.12688/f1000research.6555.1

First published: 29 May 2015, 4:134 https://doi.org/10.12688/f1000research.6555.1 


\section{Introduction}

Research resources, defined here as the reagents, materials, and tools used to produce the findings of a study, are the cornerstone of biomedical research. However, as has long been bemoaned by database curators and documented recently by Vasilevsky and colleagues, these resources are not very well identified in the scientific literature ${ }^{11}$. Vasilevsky and colleagues found that researchers did not include sufficient detail for most key research resources to allow someone to identify conclusively an antibody, a genetically modified animal, or cell lines, for example. In most cases, authors would provide insufficient metadata about the resource to conclusively identify a particular resource, e.g., a non-unique set of attributes with no catalog or stock number to specifically identify the resource used. It should be noted that the authors were, generally speaking, following the guidelines offered by the journals. Such guidelines traditionally state that one should include the company name and city in which it was located. Further, even when uniquely identifying information was provided (e.g., a catalog number for a particular antibody), the vendor may have gone out of business or the particular product may no longer be available. Given that in these cases a human cannot find which resources were used, an automated agent, such as a search engine or text mining tools will also not be able to identify the resources.

Because the current practices for reporting research resources within the literature are inadequate, non-standardized and not optimized for machine-based access, it is currently exceedingly difficult to answer very basic questions about published studies such as "What studies used the transgenic mouse I am interested in?" These types of questions are of interest to the biomedical community, which relies on the published literature to identify appropriate reagents, troubleshoot experiments, and aggregate information about a particular organism or reagent to form hypotheses about mechanism and function. Such information is also critical to funders who paid a research group to generate a particular tool or reagent and the resource providers, both commercial and academic, who would like to be able to track the use of these resources in the literature. Beyond this basic utility, identification of the particular research resource used is an important component of scientific reproducibility or lack thereof.

The Resource Identification Initiative (RII) is laying the foundation of a system for reporting research resources in the biomedical literature that will support unique identification of research resources used within a particular study. The initiative is jointly led by the Neuroscience Information Framework (NIF; http://neuinfo. org) and the Oregon Health Science University (OHSU) Library, data integration efforts occurring as part of the Monarch Initiative, http://www.monarchinitiative.org), and with numerous community members through FORCE11, the Future of Research Communications and e-Scholarship, a grassroots organization dedicated to transforming scholarly communication through technology. Since 2006, NIF has worked to identify research resources of relevance to neuroscience. The OHSU group has long-standing ties to the model organism community, which maintains databases populated by curating the literature and contacting authors to add links between model organisms, reagents, and other data. In a 2011 workshop (see https:// www.force11.org/node/4145) held under the auspices of the Linking Animal Models to Human Diseases (LAMHDI) consortium, various stakeholders from this community drafted recommendations for better reporting standards for animal models, genes, and key reagents.
The RII initiative was launched as a result of two planning meetings building off of the recommendations of the LAMHDI workshop. The first was held in 2012 at the Society for Neuroscience meeting with over 40 participants comprising editors, publishers and funders (sponsored by INCF; http://incf.org). This meeting outlined the problem of incomplete identification of research resources within papers, and the need for a computational solution for identifying and tracking them in the literature. Recognizing that any solution needed to work for both humans and machines, three broad requirements were identified: 1) the standard should be machineprocessable - that is, designed for search algorithms, in addition to human understanding; 2) the information should be available outside the paywall, so that search algorithms and humans have free access to the information across the biomedical literature; and 3) the standard should be uniform across publishers, to make uptake and usage easier for both human and machine.

A follow-up workshop at the NIH (https://www.force11.org/ node/4857) was held in June of 2013 to gain agreement from this stakeholder group for the design of a pilot that would explore solutions for this problem. A working group, the Resource Identification Initiative, was established through FORCE11, comprised of publishers, journal editors, antibody manufacturers and distributors, biocurators, software tool developers, and foundations. Based upon agreements garnered at the June 2013 meeting, the RII designed a pilot project to test implementation of a system for authors submitting manuscripts to identify research resources through the use of a unique identifier, termed a Research Resource Identifier or RRID.

\section{Pilot project overview}

The pilot project has focused on a limited number of resources antibodies, software tools/databases, and model organisms. These three resource types were chosen because they are a major source of variation across experiments and are used broadly across research communities. For the purposes of this pilot, a critical aspect was that a relatively complete and authoritative central registry existed that could issue an accession number, as Genbank does for gene sequences. To gain broad agreement amongst publishers and editors who were concerned about the potential burden on authors and staff, it was agreed that participation in the pilot project would be voluntary for authors with participation not representing a condition of acceptance for publication. The pilot project was also designed to have minimal requirements for publishers such that modification of manuscript submission systems was not required.

The pilot project was originally designed to run for 6 months, with each of the participating journals agreeing to participate for at least 3 months. The goal was to ensure a large enough sample to understand author behavior: could they and would they do the task, to test the sufficiency of the infrastructure, and to obtain a sufficiently large dataset to explore and demonstrate the utility of RRIDs. Over the minimum 3-month window, each partner journal would request that authors supply RRIDs in a standard format as a citation to indicate the use of any of these three types of research resources. To be as unambiguous as possible, authors were to include the RRIDs in the text of the materials and methods, but not in the introduction or discussion sections. The RRID syntax comprises an accession number assigned by the authoritative database with the prefix "RRID:” prepended (e.g., RRID:AB_2298772 
for an antibody). We also requested that closed-source journals include RRIDs in the keyword field as this field is available for indexing in PubMed outside of paywalls. The journals were given flexibility for when and how they wanted to ask authors for these identifiers, namely, at time of submission, during review, or after acceptance. They were not required to modify their instructions to authors or their submission systems. The RII team would be responsible for preparing appropriate materials for requesting RRIDs and for establishing a central portal where these identifiers could be obtained. The RII team also agreed to establish a help desk to assist the authors if they encountered any difficulties.

The pilot project was designed to address four key questions. A set of evaluation criteria was designed for each question:

1. Compliance: Would authors be willing to add resource identifiers to their publications and register new resources in the system? Compliance was evaluated by examining the number of submissions to the participating journals, the rate of author compliance in providing RRIDs, the number of new resources registered, and direct feedback from authors.

2. Accuracy: Could authors add these identifiers accurately or would additional editorial or staff oversight be necessary? Accuracy was measured by a quantitative analysis of RRID accuracy by RII curators.

3. Identifiability: Would the use of RRIDs improve our ability to identify resources in the literature? Identifiability was measured by assessing the number of catalog numbers preand post-pilot in the journals that participated.
4. Utility: Will RRID's be useful to the scientific community? Can the RRID's as constructed be used to identify all studies that use a particular research resource? To encourage the development of applications, the data set is being made freely available so that third parties can develop tools to work with RRIDs.

The pilot began in February 2014, with over 25 journals participating. Journals that sent a letter to authors at some stage of the review process included: Journal of Neuroscience, Brain and Behavior, Journal of Comparative Neurology, Brain Research, Experimental Neurology, F1000Research, PeerJ, Journal of Neuroscience Methods, Neurobiology of Disease, and the Frontiers group of journals. One journal, Neuroinformatics, chose to add the RRIDs to all manuscripts before asking authors to do this. Journals in the Elsevier and BMC groups were participants based upon updates to their instructions to authors. Because of the success of the project, it was subsequently extended and is still active as of this writing. The number of journals participating has expanded, and now includes PLoS Biology and PLoS Genetics as well as multiple immunology journals in the Elsevier family. A list of the participating journals is available on the Force11 website (https://www.force11.org/RII/SignUp).

\section{Workflow}

One of the prime requirements of the pilot project was to make it as easy as possible for authors to obtain the appropriate identifiers and insert them correctly into their manuscripts. As noted above, the three research resources were chosen because each was covered by an authoritative database (Table 1) that assigned unique IDs and a standard set of metadata to each. However, as can be seen by the length of the list in Table 1, authors could potentially be required to visit several databases to obtain the appropriate identifiers.

Table 1. Source databases and registries included in the RII portal. Each database has a weekly or monthly scheduled frequency of update and all new data is released weekly. If available, data from both model organism authorities is served as well as the list of strains available via particular stock centers. In most cases the stock centers maintain a link between the genotype and the stock center animal identifier. Scheduling and total data count information can be accessed via DISCO (http://disco.neuinfo.org/webportal/dataPipelineViewStatus.do?id=nlx_154697-1).

\begin{tabular}{|l|l|l|}
\hline Resource name & Resource content & Database Identifier \\
\hline ZIRC, Zebrafish Resource Center & Zebrafish Stocks & RRID:nif-0000-00242 \\
\hline ZFIN, Zebrafish Information Network & Zebrafish Nomenclature & RRID:nif-0000-21427 \\
\hline RGD, Rat Genome Database & Rat & RRID:nif-0000-00134 \\
\hline $\begin{array}{l}\text { CGC, Caenorhabditis Genetics Center } \\
\text { WormBase }\end{array}$ & Worm Stocks & RRID:nif-0000-00240 \\
\hline $\begin{array}{l}\text { IMSR, International Mouse Strain Resource } \\
\text { Center }\end{array}$ & Worm Nomenclature & RRID:nif-0000-00053 \\
\hline $\begin{array}{l}\text { BDSC, Bloomington Drosophila Stock Center } \\
\text { MGI, Mouse Genome Informatics }\end{array}$ & Fly Stocks & RRID:nif-0000-09876 \\
\hline BCBC, Beta Cell Biology Consortium & Mouse Nomenclature & RRID:nif-0000-00096 \\
\hline antibodyregistry.org, Antibody Registry & Mouse stocks & RRID:nlx_144143 \\
\hline SciCrunch Registry & Antibodies & RRID:nif-0000-07730 \\
\hline
\end{tabular}


To simplify this process, we utilized the data aggregation services of the NIF, provided through a platform known as SciCrunch, to establish a Resource Identification Portal (http://scicrunch.org/resources; Figure 1). The portal provided a unified query across these different databases and displayed the results in a common format. The portal allows search on various facets such as resource name, catalog number, etc. There is a 'cite this' link that provides the citation as it should be reported in the paper. The citation generally includes not just the RRID, but a set of appropriate metadata that would identify the vendor and catalog number as well, for example: A polyclonal antibody against tyrosine hydroxylase (TH) (Chemicon, Cat. AB1542, RRID:AB_90755).

SciCrunch was built based on the extensible Neuroscience Information Framework platform described previously (12,13, RRID: nif-0000-25673), and the portal infrastructure for RII was developed under an award from NIDDK to create a dkNET portal (RRID: nlx_153866), while the customization of the portal was done by Monarch staff. The data is aggregated from the SciCrunch tool registry, the antibody registry, as well as the model organism community databases and stock centers (Table 1). The data infrastructure allows curators to keep indexes synchronized with the source databases by using an automated crawling engine and new data are released on a weekly basis. All open data from each of these databases is available to download from the source sites, where update frequencies are listed.

The instructions to authors in all cases were consistent, that is, the same set of instructions was provided to each author. For antibodies, we only required authors to identify primary antibodies and not secondary or tertiary complexes. For tools and databases, we focus on freely available tools, generally supported through public funding and not commercial tools. Authors were asked to insert the correct citation for the resource into the text of the materials and methods section and in the keywords. A help desk was established by the RII working group that provided help if an author encountered difficulty. In most cases, requests were handled in less than 24 hours.

If a resource was not found via the portal, authors were given the option of submitting the resource to obtain an identifier. For antibodies and software/databases, which are found in databases maintained within the NIF, submission was handled through the Resource Identification Portal. For model organisms, the author was referred to the authoritative model organism database. All new submissions were curated by their respective databases and the data was pulled back into the RII portal weekly so that authors could see their newly registered antibodies or software tools in about a week.

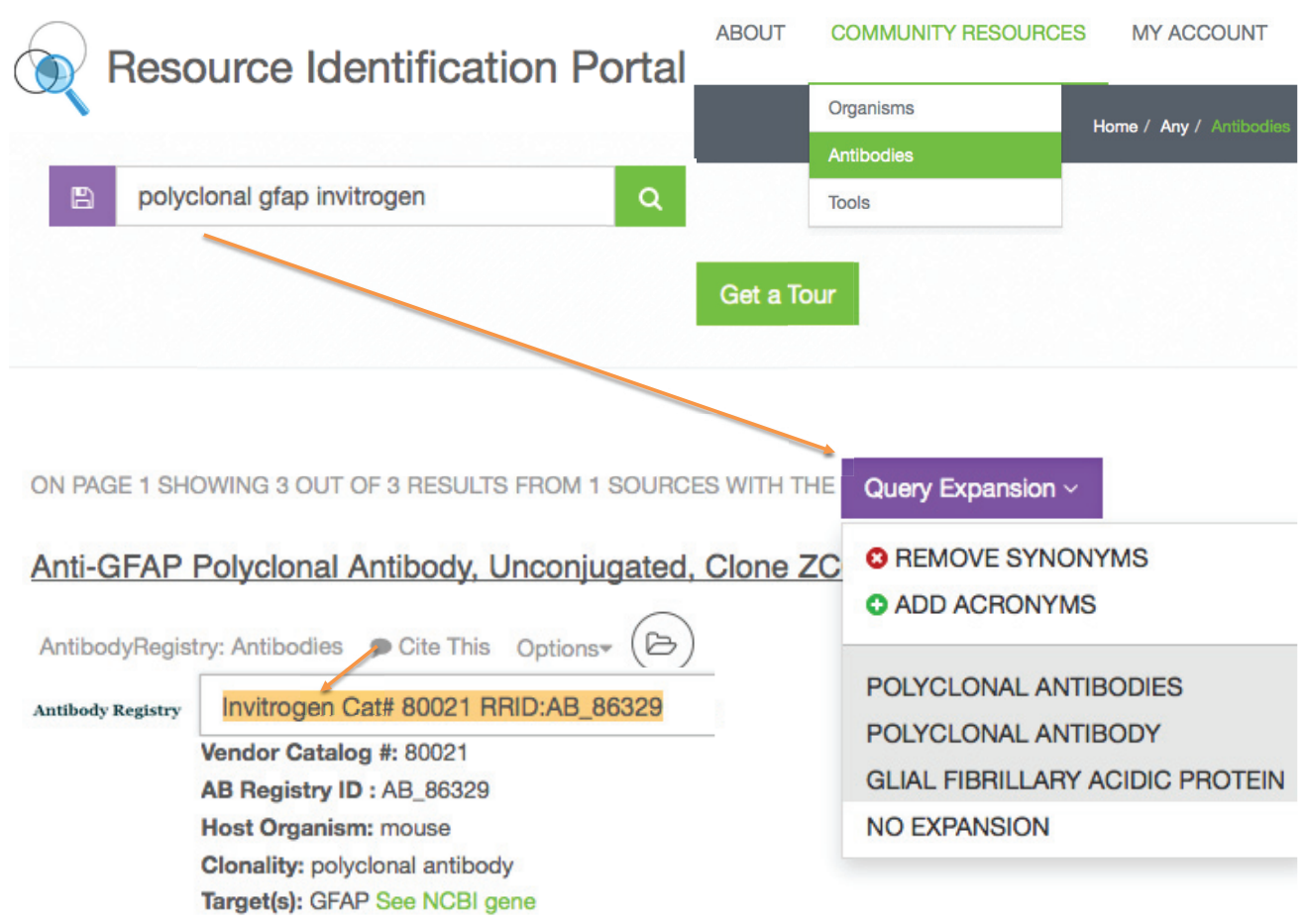

Figure 1. Resource Identification Initiative portal where authors are asked to find the Research Resource Identifiers (RRIDs) for inclusion in the methods section of their publication. The workflow for authors is to select their resource type (see community resources box), type in search terms (note, the system attempts to expand known synonyms to improve search results) and open the "Cite This" dialog box. The dialog shown here displays the Invitrogen catalog number 80021 antibody with the RRID:AB_86329. The authors are asked to copy and paste this text into their methods sections. 


\section{Results}

The first RRIDs began appearing in the literature in April of 2014. Although the first paper was identified through PubMed, the majority of papers were found via Google Scholar by searching for "RRID". Google Scholar, unlike PubMed, appears to search the full text of articles, as it returns snippets of text from the materials and methods containing the RRIDs (for example see Figure 2). A search in PubMed returns very few papers, indicating that publishers were not including the RRIDs outside of the pay-wall. As these papers start to appear in PubMed Central where there is full text search, it should be possible to find papers for RRIDs through the National Library of Medicine. Google Scholar possesses the advantage in that it obtains papers without an embargo period and makes them available for search, unlike PubMed Central. We therefore present statistics and analyses in this paper based upon Google Scholar.

Search via Google Scholar reveals that the RRID prefix is not a unique string, but is an acronym for several entities, mostly commonly the Renal Risk in Derby clinical study (for example 9). To return examples of RRIDs requires the use of additional filters, e.g., restricting search to the years 2014 and later. The combination of the RRID prefix with the resource accession number is unique however, in that searching for a particular RRID, for example RRID:AB 90755 returns only papers that use this research resource (Figure 2).

To address the aims of the pilot project, we tracked the use of RRIDs in published papers and journals. We performed an in-depth analysis of the first 100 papers found through Google Scholar that reported RRIDs. For each paper, we examined the methods section to determine whether RRIDs were correctly cited - that is that they pointed to the correct resource (= true positives), and whether all research resources that should have been identified by an RRID were appropriately cited (= true negatives). The total number of research resources reported in the first 100 papers of the Resource Identification Initiative was determined by manual inspection of each paper by two independent people. A Google Scholar alert was used to track all new papers that contained the term RRID. Each paper was downloaded and examined for the snippets of text surrounding research resources (methods or data use sections). Each snippet of text surrounding the RRID was copied and pasted into a shared document (Supplemental table 1). The RRID was then checked against the scicrunch resolving service (for example https://scicrunch.org/resolver/RRID:AB_262044) to determine what the source database lists about the resource. Information was compared in the following way: if the vendor/catalog number was present in the snippet and these matched the resolver data, we considered that the record was marked accurate. If no information about the catalog number was present in the paper, but the antibody target or clone number matched then the record was also marked accurate, but if the vendor/catalog number information was different from the database record or no other information could be found about the reagent, then the record was marked inaccurate. The total accuracy is primarily therefore based on the catalog number match and importantly does not reflect any upstream problems with organism or reagent identification inside of the laboratories themselves.

\begin{tabular}{|c|c|c|c|}
\hline & ID & Name & xUsed \\
\hline RRID:AB_90755 & RRID:nif-0000-30467 & ImageJ & 16 \\
\hline \multirow[b]{2}{*}{4 results $(0.04 \mathrm{sec})$} & RRID:nlx_153890 & MATLAB & 10 \\
\hline & RRID:nif-0000-00343 & SPM & 6 \\
\hline \multirow{7}{*}{$\begin{array}{l}\text { A general principle governs vision-dependent dendritic patterning of retinal gangli } \\
\text { HP Xu, JH Sun, N Tian - Journal of Comparative Neurology, } 2014 \text { - Wiley Online Library } \\
\ldots \text { A polyclonal antibody against tyrosine hydroxylase (TH) (Chemicon, Temecula, CA, AB } 1542, \\
\text { RRID: AB_90755) was used to label TH-positive dopaminergic amacrine cells in the retina. ... and } \\
\text { western blotting of PC12 cells (single 60-kDa banc), RRID: AB_90755, ... } \\
\text { Related articles All } 3 \text { versions Cite Save } \\
\text { The orexinergic neurons receive synaptic input from C1 cells in rats } \\
\text { G Bochorishvili, T Nguyen, MB Coates... - Journa of ..., } 2014 \text { - Wiley Online Library } \\
\ldots \text { TH mouse anti-sheep antibodv (AB1542: Millipore: RRID:AB_90755) was raised against native } \\
\text { TH from rat pheochromocytoma. As reported by Nillipore, this antibody recognizes a protein } \\
\text { of approximately } 60 \mathrm{kDa} \text { by western blot of mouse brain lysate. ... } \\
\text { Cite Save }\end{array}$} & RRID:AB_2298772 & Anti-NeuN antibody & 5 \\
\hline & RRID:AB_839504 & Anti-Iba-1 Polyclonal & 4 \\
\hline & RRID:AB_90755 & Anti-TH antibody & 4 \\
\hline & RRID:nif-0000-10294 & Neurolucida & 4 \\
\hline & RRID:nif-0000-23420 & SynapseWeb Recns & 4 \\
\hline & RRID:AB_10013382 & GFAP antibody & 3 \\
\hline & RRID:AB_10049650 & AlexaF.488 (A21206) & 3 \\
\hline \multirow{8}{*}{$\begin{array}{l}\text { Catecholaminergic connectivity to the inner ear, central auditory, and vocal motor circuitry in th } \\
\text { plainfin midshipman fish porichthys notatus } \\
\text { PM Forlano, SD Kim, ZM Krzyminska... - Journal of ..., } 2014 \text { - Wiley Online Library } \\
\text {... The primary antibodies and dilutions were as follows: mouse anti-tyrosine hydroxylase (TH; } \\
\text { 1:1,000; Millipore/Chemicon MAB318, RRID: AB_2201528, Temecula, CA), sheep anti-TH } \\
\text { (1:3,000; Millipore/Chemicon AB1542, RRID: AB_90755), and mouse anti-hair cell HCS-1 (1 ... } \\
\text { Related articles All } 6 \text { versions Cite Save } \\
\text { GABAergic and Glutamatergic Efferents of the Mouse Ventral Tegmental Area } \\
\text { SR Taylor, S Badurek, RJ DiLeone... - Journal of ..., 2014 - Wiley Online Library } \\
\ldots \text { Antibody Characterization Sheep anti-tyrosine hydroxylase, polyclonal According to the } \\
\text { manufacturer (Millipore, Billerica MA:Cat. No. AB1542, RRID: AB_90755), the primary antibody } \\
\text { was raised against native tyrosine hydroxylase (TH) from rat Page } 8 \text { of } 70 \text { John Wiley \& Sons ... } \\
\text { Cited by } 2 \text { Related articles All } 3 \text { versions Cite Save }\end{array}$} & RRID:AB_143165 & AlexaF.488 (A11008) & 3 \\
\hline & RRID:AB_2079751 & ChAT Antibody & 3 \\
\hline & RRID:AB_390204 & TH Antibody & 3 \\
\hline & RRID:AB_477585 & Tubulin Antibody & 3 \\
\hline & RRID:AB_477627 & Vimentin Antibody & 3 \\
\hline & RRID:nif-0000-00304 & FreeSurfer & 3 \\
\hline & RRID:nif-0000-10474 & R Project & 3 \\
\hline & RRID:nif-0000-31484 & SAS Project & 3 \\
\hline
\end{tabular}

Figure 2. RRIDs found in the published literature. A. Google scholar result for the anti-tyrosine hydroxylase antibody RRID (9/2014; http://scholar.google.com/scholar?q=RRID:AB_90755). B. Shows the most used RRIDs in the first 100 papers, by number of papers using the identifier. All data is available in Supplementary Table 1 and all identifiers can be accessed in Google Scholar (see also Supplemental Table 1). 
The results of the analysis are summarized in Table 2 . The spreadsheet containing the full analysis is available as a supplementary data file. The first 100 papers were published in 15 journals and included 630 RRIDs reported by authors. The bulk of the identifiers (572) came from two journals, the Journal of Comparative Neurology and the Journal of Neuroscience, as these two journals were first to participate both starting the pilot in early February.

\section{Outcome \#1: Compliance}

As of March 1, 2015 there were 312 papers published with at least one RRID, from 44 unique journals (Supplementary files show the updated list of journals and a count for each) indicating that hundreds of authors have participated in the pilot project even though it is voluntary. Informal feedback from the editors and authors via help requests and other correspondence indicates that authors who are attempting to find RRIDs are supportive of the aims of the project and readily able to find the correct RRIDs.

Authors were willing to add resources to the registries if they were not available. Since the project began, over 200,000 antibodies from vendors - both solicited and unsolicited - and at least
200 from individual authors were added to the Antibody Registry (http://antibodyregistry.org). In cases where antibodies are sold by government-led projects such as NeuroMab from UC Davis, antibody identifiers have been included in the antibody manufacturer's web site. Many of the additions were secondary antibodies, which were not part of the project but authors felt that they should also be identified. In one representative example, Jackson ImmunoResearch was contacted by several authors and submitted their full catalog to the Antibody Registry, allowing authors to identify secondary antibodies. Additionally, there were over 100 software tools and databases registered. Many were for common commercial statistical tools (e.g., SPSS, GraphPad), technically out of scope for the project, but authors did not make the distinction between commercial and non-commercial tools. Figure 2 shows the most common tools identified by RRID in papers from the first 100 papers. Commercial tools such as MATLAB, SAS and GraphPad were cited along with ImageJ and FreeSurfer. The most common antibody was NeuN from Millipore. These same resource identifiers have continued to be very highly cited in subsequent papers, with ImageJ cited in 42 papers and the NeuN antibody cited in 8 papers (Google Scholar March 17, 2015). A comparison of added

Table 2. Summary of journal practices. Journals that added only the instructions to authors are not included in this table (for example BMC). The compliance rate was by far the lowest with only instructions to authors; this hovered between 1 and $15 \%$ when authors were asked by blanket mailing in addition to the instructions to authors (A letter to authors from the editor, when this letter included several pages of instructions. Compliance was lowest in Brain Research and Journal of Neuroscience Methods) and very high if the editorial staff asked authors directly or suggested identifiers for their manuscript. For two journals this was further facilitated by a special section in the papers that described tools, Journal of Comparative Neurology and Neuroinformatics both contain sections that normally describe antibodies and software tools, respectively.

\begin{tabular}{|c|c|c|c|c|c|}
\hline Journal & Submission & Review & Acceptance & Compliance & Notes \\
\hline $\begin{array}{l}\text { Journal of } \\
\text { Neuroscience }\end{array}$ & $\begin{array}{l}\text { Letter } \\
(1175)\end{array}$ & $\begin{array}{l}\text { Letter } \\
(163)\end{array}$ & Letter (26) & $\sim 12 \%$ & $\begin{array}{l}\text { Asking at different stages has no effect on rate of } \\
\text { compliance }\end{array}$ \\
\hline $\begin{array}{l}\text { Journal of } \\
\text { Comparative } \\
\text { Neurology }\end{array}$ & $\begin{array}{l}\text { Working } \\
\text { with Author }\end{array}$ & $\begin{array}{l}\text { Working } \\
\text { with Author }\end{array}$ & $\begin{array}{l}\text { Working } \\
\text { with Author }\end{array}$ & $>90 \%$ & $\begin{array}{l}\text { Published an editorial and has a history of proper antibody } \\
\text { identification back to } 2006\end{array}$ \\
\hline $\begin{array}{l}\text { Brain and } \\
\text { Behavior }\end{array}$ & & & $\begin{array}{l}\text { Letter } \\
(\sim 100)\end{array}$ & $\sim 25 \%$ & $\begin{array}{l}\text { Letters started to be sent out in April 2014, at times the } \\
\text { editor followed up with authors, did not keep exact records }\end{array}$ \\
\hline Neuroinformatics & & & $\begin{array}{l}\text { Staff looks } \\
\text { up data }\end{array}$ & $100 \%$ & $\begin{array}{l}\text { Journal has a section for tools used in the study, which now } \\
\text { includes RRIDs, several papers incorporated RRIDs prior to } \\
\text { staff intervention }\end{array}$ \\
\hline F1000Research & & & Letter $(\sim 50)$ & $12 \%$ & Approximate figure from editor \\
\hline Brain Research & & $\begin{array}{l}\text { Letter } \\
(671)\end{array}$ & & $1 \%$ & $\begin{array}{l}\text { Authors receive automatically generated letters with } \\
\text { multiple instructions, including RII guidelines. Authors are } \\
\text { asked to incorporate RRIDs or database identifiers (overall } \\
\text { compliance } 1 \% \text {; for RRIDs }<1 \% \text { ). }\end{array}$ \\
\hline $\begin{array}{l}\text { Journal of } \\
\text { Neuroscience } \\
\text { Methods }\end{array}$ & & $\begin{array}{l}\text { Letter } \\
(314)\end{array}$ & & $1 \%$ & $\begin{array}{l}\text { Authors receive automatically generated letters with } \\
\text { multiple instructions, including RII guidelines. Authors are } \\
\text { asked to incorporate RRIDs or database identifiers (overall } \\
\text { compliance } 1 \% \text {; for RRIDs }<1 \% \text { ). }\end{array}$ \\
\hline $\begin{array}{l}\text { Neurobiology of } \\
\text { Disease }\end{array}$ & & $\begin{array}{l}\text { Letter } \\
\text { (291) }\end{array}$ & & $3 \%$ & $\begin{array}{l}\text { Authors receive automatically generated letters with } \\
\text { multiple instructions, including RII guidelines. Authors are } \\
\text { asked to incorporate RRIDs or database identifiers (overall } \\
\text { compliance 3\%; for RRIDs } 2 \% \text { ). }\end{array}$ \\
\hline $\begin{array}{l}\text { Experimental } \\
\text { Neurology }\end{array}$ & & $\begin{array}{l}\text { Letter } \\
\text { (297) }\end{array}$ & & $3 \%$ & $\begin{array}{l}\text { Authors receive automatically generated letters with } \\
\text { multiple instructions, including RII guidelines. Authors are } \\
\text { asked to incorporate RRIDs or database identifiers (overall } \\
\text { compliance } 3 \% \text {; for RRIDs }<1 \% \text { ). }\end{array}$ \\
\hline
\end{tabular}


resources vs those reported in the first 100 papers indicates that the Registries already listed the majority of research resources in each of these categories, as the number of new resources added for this set represented only $10 \%$ of the total reported resources.

\section{Outcome \#2: Accuracy}

A major concern of the publishers and editors was whether or not authors could retrieve RRIDs accurately and whether significant editorial oversight would be necessary for quality control (see workshop outcome documents at https://www.force11.org/node/4857). As shown in Table 3, authors of the first 100 papers were $96 \%$ accurate when reporting resource identifiers - that is, they used the correct identifier as determined by comparing the metadata supplied in the article with that in the database, accessible via a resolving service (see http://scicrunch.com/resolver/RRID:AB_262044). Authors did not create RRIDs for resources they were either unable to find, or were not in the databases in 34 clear-cut cases. This constitutes a $5.4 \%$ false negative rate. In cases where authors tried to identify research resources they tended to include identifiers for over $90 \%$ of them.

A total of 24 errors were found in the 630 RRIDs analyzed. For antibodies, 15 antibodies were incorrectly identified (3.8\% error rate). Inspection of these errors, showed that: three errors were copy/paste mistakes where authors mixed up the combination of catalog number and identifier for resources actually used in their paper; three errors resulted from identifiers missing a digit at the end of the ID (for example, "Swant, catalog \#6B3, RRID: AB_1000032" should have been named RRID: AB_10000320); one error involved reporting a reference PMID instead of the resource identifier. The apparent cause of the other eight antibody errors was not possible to determine. For organisms, seven errors were made (13.2\% error rate). All of these errors involved mice for which authors used the appropriate gene or allele identifier from Mouse Genome Informatics, MGI, rather than the stock number or genotype identifying the organism. The allele ID is better than no information, but it is not

Table 3. Summary statistics for the total number of research resources reported in the first 100 papers of the Resource Identification Initiative. Counts were ascertained by two curators going through every paper with at least one RRID, and \%Error was calculated when the information surrounding the identifier reported in the paper or the identifier did not match the data from the resolving service for each entity. For a complete list of resources, including links to the resolver, see Supplementary table 1.

\begin{tabular}{|r|r|r|}
\hline antibody & Counts & \%Error \\
\hline organism & 549 & $3.56 \%$ \\
\hline software/database & 128 & $13.21 \%$ \\
\hline total & $\mathbf{6 3 0}$ & $0.78 \%$ \\
\hline & & $\mathbf{3 . 8 1} \%$ \\
\hline no ID in paper & 34 & $5.40 \%$ \\
\hline pulled new ID & 47 & $7.46 \%$ \\
\hline
\end{tabular}

sufficient for identifying the animal used as the same allele may be inserted into different mice of various backgrounds and with other alleles, and those mice may have different characteristics. It should also be noted that authors using the MGI database (up to October 2014), which maintains the authoritative mouse database, would be given a set of MGI identifiers for genes and alleles, but not genotypes. This shows that authors likely went to MGI to obtain their identifiers, but were not able to find the genotype information and substituted the allele ID. Currently MGI contains a Google site search that now searches the genotype information for all mice suggesting that authors of newer papers can now also find the genotype information more easily at MGI. They have also created a tutorial for how to obtain a genotype identifier that has been posted on the SciCrunch pages. The ideal situation is for MGI, the trusted authority, to point authors to the proper genotype information. The fewest errors were made in identifying software tools and databases, with only one mistake from 129 total $(0.8 \%)$. The mistake was made as the author apparently used an antibody identifier instead of a tool identifier. From the relatively low error rate, it appears that authors are able to perform the task accurately, at least for software tools, databases, and antibodies.

\section{Outcome \#3 Identifiability}

An outcome of this study was to determine if the use of RRIDs in the literature increased the identifiability of research resources. As shown in Figure 3, when authors were asked by their editors to provide RRIDs, regardless of their compliance with the RII project, the identifiability of research resources significantly increased. We

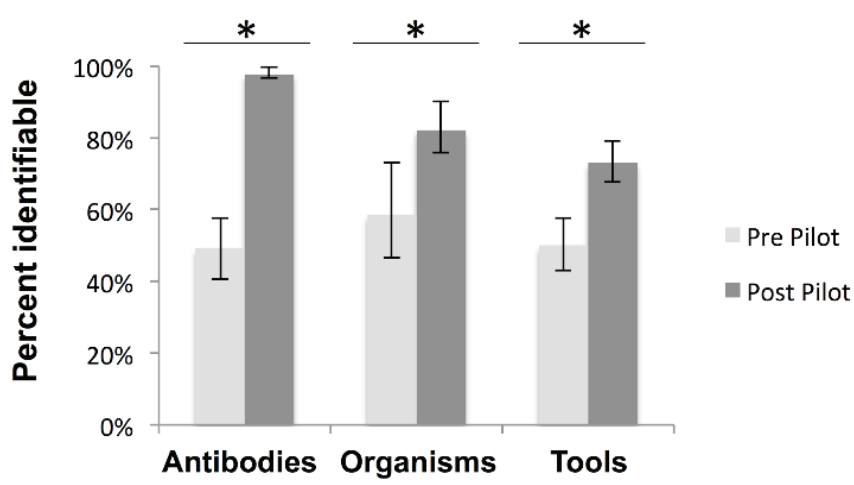

Figure 3. Resource identification pre- and post-RIl pilot study. Identifiability of the three resource types was determined in journal articles that reported RRIDs (post-pilot), and in articles from the same journals before the pilot started (from approximately January-March 2013, pre-pilot). Resources (primary antibodies, organisms, and tools) were considered identifiable if they contained an accurate RRID or by using the same criteria as described in Vasilevsky et al., 2013 (PMID: 24032093). For software and databases (which were not previously analyzed), these resources were considered identifiable if they contained an RRID or reported the manufacturer and version number. The total number of resources for each type is: primary antibodies pre-pilot, $n=140$; primary antibodies post-pilot, $n=433$; organisms pre-pilot, $n=58$; organisms post-pilot, $n=128$; tools prepilot, $n=176$; tools post-pilot, $n=246$. The $y$-axis is the average for each resource type. Variation from this average is shown by the bars: error bars indicate upper and lower 95\% confidence intervals. Asterisks indicate significant difference by a z-score greater than 1.96. 
calculated the percentage of identifiable research resources in the same journals, just before the pilot project and after. The reporting of research resources pre-pilot was consistent with findings from the 2013 study (Vasilevsky et al., 2013), in that roughly 50-60\% were found to be identifiable. But when asked by their editors, researchers used identifying information in $80-90 \%$ of research resources, showing that they presumably had the data available, but did not put it into their papers unless prompted by communication from the editors.

\section{Outcome \#4 Utility}

Consistency of syntax and machine-processability: The ability to search all studies that used a particular research resource was a prime motivation for this pilot project. The current project had a loose definition of "machine-processable" because we did not want to impose any requirements on the publishers to modify their journal submission system for a pilot project. Thus, we opted to craft RRIDs as unique, indexable alphanumeric strings that could support use of web search engines to return papers that used a particular research resource. We specifically asked authors to place the RRIDs only in the materials and methods section, where they would normally provide identifying information for a given entity, because we wanted to track actual use of the resource and not just mentions of it.

For individual RRIDs, the approach was highly successful as is illustrated by the ability to type a particular RRID into three search engines for the biomedical literature: Google Scholar, PubMed and Science Direct and retrieve appropriate papers, e.g., RRID: AB_90755 or AB_2298772 (for Google Scholar see Figure 2). It is important to note that each of these systems will come back with different results because each search tool has different types of data about each paper. For example, ScienceDirect has a good full text search of all Elsevier content, but it does not search other publishers content. Both PubMed and Scopus search only the abstracts and return a subset of articles where authors followed instructions to add RRIDs to the keywords, but not those that are only in the methods section. Google Scholar is the most comprehensive as it appears to search full text and brings back papers that are both published and unpublished (usually these are accepted for publication, but not yet indexed by PubMed). An analysis performed in October, 2014 showed varying results from each search engine: Google Scholar returned 315 results (from 2014, 174 are true RRIDs), and ScienceDirect returned 18 (from 2014, three are RRIDs). PubMed revealed 23 papers that contained RRIDs (from 2014, all identify the resource identification initiative identifiers). Scopus returned 48 documents (from 2014, 18 are RRIDs).

The use of a unique string to retrieve RRIDs is aided by a common syntax. Thus, in our analysis of RRIDs, we also noted whether or not the RRID was correctly formed. While authors were $96 \%$ accurate in supplying identifiers, minor corrections to formatting were needed in $34 \%$ of RRIDs (66\% accuracy). The most common variant was the addition of extra spaces (RRID:AB_90755 vs RRID: AB_90755), other common variants were failure to include the RRID prefix (RRID as table header), using various symbols or spaces in the identifier, or splitting up the RRID prefix and identifier in a table. These practices make it more difficult to identify the RRID through text search alone, and require some additional natural language processing to identify. We note, however, that the search algorithms are improving. Whereas in June, Google Scholar could not recognize an RRID that included a space after the colon, e.g., RRID:AB_\#\#\#\#, by October these types of variants were retrieved. Such minor errors could also be avoided by developing authoring system upgrades that directly identify RRIDs and insert the proper citations into the manuscript.

To promote the development of 3rd party tools around RRID's, we created a resolver service for RRIDs using SciCrunch. Typing http:// scicrunch.com/resolver/RRID:AB_90755, will resolve to a landing page with meta-data on a particular entity. The resolving service allows applications to make use of RRIDs to, for example, enhance articles with RRIDs by providing additional information about the entity and a link to relevant articles and resources. For instance, Elsevier has released their antibody application, which displays antibody meta-data in the right hand side panel, next to the article (see the screen shot below (Figure 4) for 8: http://www.sciencedirect.com/science/article/pii/S0306452214008458). The reader can browse through antibodies referred to in the article, view complete records in antibodyregistry.org and access additional information via direct links to GenBank, ZFIN and other relevant databases. The application also recommends three most relevant articles published in Elsevier journals that refer to the same antibody. The application is freely available on ScienceDirect.

Publication practices: Non-open access journals were asked to add RRIDs to publication keywords, but our initial findings suggest that this practice was not being consistently followed. Only 23 papers out of 41 total (as of Oct 20, 2014) were accessible in PubMed. Additionally it should be noted that in two cases, identifiers were removed at typesetting after the initial online version of the manuscript was published with the RRIDs. These identifiers were removed not only from the manuscript, but also from PubMed keywords. Although this was reversed when noted by the working group, this demonstrates that successful implementation requires knowledge of and agreement by the publishers at all steps.

\section{Discussion}

The pilot RRID project has been highly successful in demonstrating the utility of a system to aid in identification of these three research resources in the literature. We showed that authors were willing to adopt new styles of citation for research resources that promoted more accurate identification of research resources used in a study, and that were more amenable to machine-based identification. To date, RRIDs have appeared in over 300 papers from 40 journals. With one exception (the Journal of Neuroscience), journals have continued their request for RRIDs beyond the initial 3-month pilot project and new journals have signed up beyond the initial set that started the project. We believe that the success of the project was due to the extensive pre-planning that involved the publishers and the editors, the limited scope of the initial request, and the recognized need by researchers for better and more useful reporting standards for research resources.

The load on curation staff with participating journals has been minimal and the initial portal prototype appears reasonable for the majority of authors to find their resource identifiers. With $>10,000$ searches in the RII portal, there were approximately 100 help questions. 


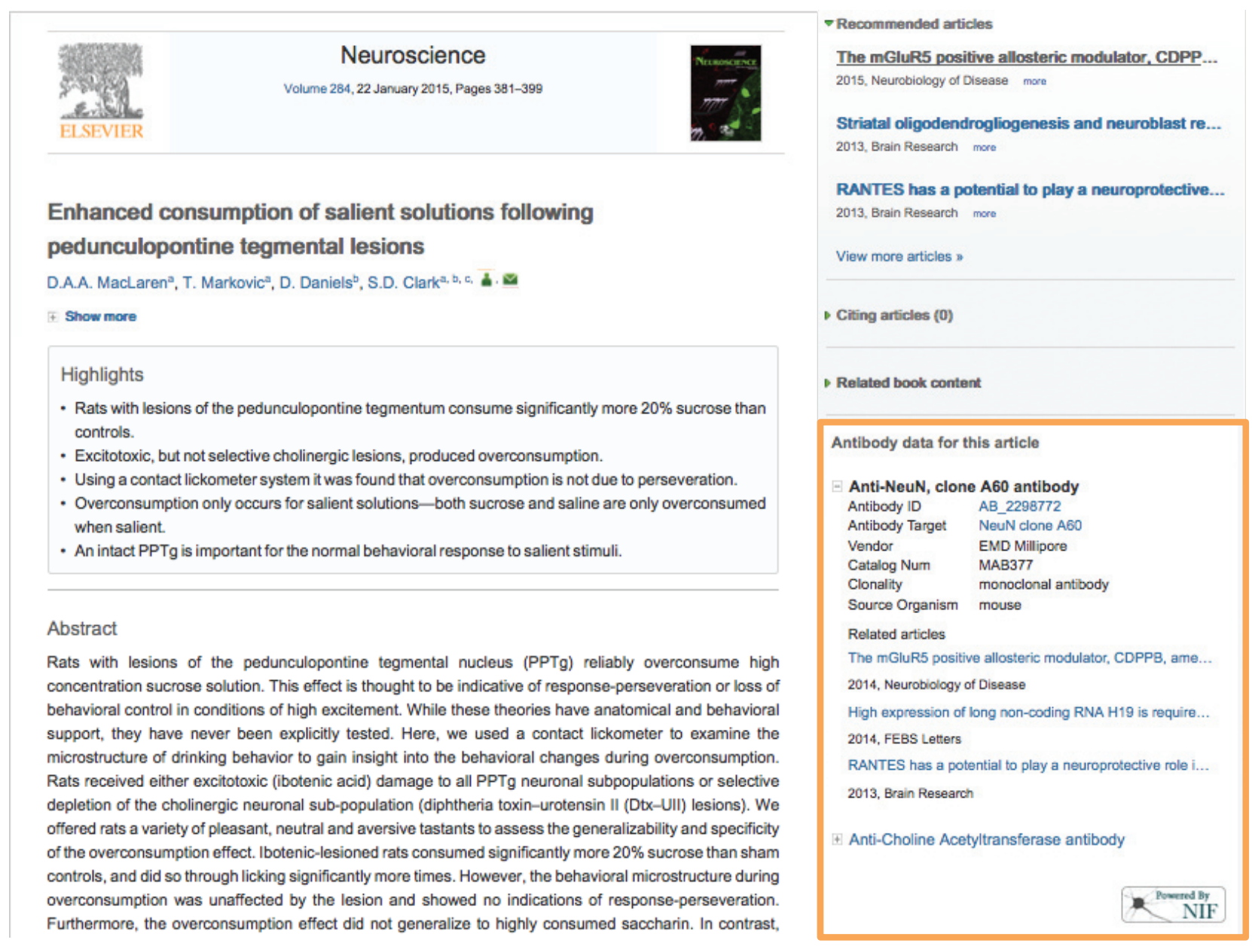

Figure 4. Representation of the "Antibody data for this article" application developed by Elsevier to enhance articles on ScienceDirect. The application is available in 206 articles in 19 journals (more information can be found at: http://www.elsevier.com/about/content-innovation/ antibodies).

Many of these questions were about scope, i.e., whether a particular research resource should be identified. Others were for assistance in finding a resource or guidance in adding a resource not yet contained in the community authorities. While this is not a large number, it is also not insignificant, particularly as the project expands, and certainly points out the need for specific help functions.

Given the relative completeness of the registries and the rapid advance of machine-learning based techniques for entity recognition, we can envision a semi-automated system that assists the author in supplying correct IDs. We have already improved our ability to detect digital research resources in the literature using machine learning (Ozyurt et al., submitted). In this system, machine learning is used to identify software tools and databases in text and compare the information to Registry listings. The development of such functions would allow the development of recommender systems for authors and automated fact checkers for journal staffs.
Why unique identifiers?

Unique identifiers serve as a primary key or "social security number" for identifying a given entity, and providing the ability for search engines to parse them is paramount. For search engines, unique identifiers are simple methods for disambiguating entities with similar names. For identifiers to function in this mode, they need to be unique - that is, the same ID should not point to two different entities, and they need to be persistent - that is, they need to outlive the entity itself. They also need to be at least minimally machineprocessable. While many authors supplied identifying information like the catalog number for an antibody supplied by the vendor, or the official strain nomenclature supplied by the IMSR for a mouse, neither of these served the required functions. A catalog number is not a unique identifier, but rather a useful way for vendors to identify their products. If the same antibody is sold by different vendors, it will have a different catalog number. If the same antibody is sold in different aliquots, it may also have a different catalog number. When 
the antibody is no longer available, the catalog number may disappear, or in some cases be recycled for use with another antibody. All of these features are undesirable in an identifier system. The Antibody Registry, in contrast, was specifically designed to supply useful and stable identifiers for antibodies and not as a commercial source of antibodies. Similarly, the strain nomenclature developed by the Jackson Laboratory, with its superscripts and special characters, is useful for human curators to identify a particular strain, but causes hiccups in most search engines because of all of the special characters. We believe that a well curated registry is essential to the success of such a system, because of the necessity of these two functions, which currently cannot be replaced with a simple uncurated registration service. For example, we found in the registries we maintain (both software and antibodies) that authors sometimes register an entity that is found by a curator to be a duplicate.

\section{Which identifiers?}

There are many types and formats of identifiers in use today (e.g., DOIs, URIs, ARCs), each with varying amounts of associated infrastructure and use in different communities. For this project, we elected to use simple alphanumeric strings and a common syntax in the form of accession numbers issued by the authoritative community-based registries. We relied on each registry to impose the uniqueness constraint at the level of the entity, for example ensuring that there was only one mouse genotype per unique ID, and to ensure standard metadata by curating each entry. The reuse of authoritative accessions with the RRID prefix provides maximal flexibility and interoperability and minimal ID churn, whilst also provisioning for resource identification.

A frequent question regarding the RRID is why we did not use a DOI as a unique identifier instead of the Registry Accession number. Part of the reason was cultural: researchers were used to supplying accession numbers for Genbank, Gene Expression Omnibus, Protein Data Bank, etc. and understand this requirement. Part of the reason is practical: unlike DOIs, accession numbers are already available for all of the research resources to be identified in this pilot and did not require special infrastructure to resolve or cost to issue. Part of the reason is also philosophical: DOIs are for digital objects, such as individual articles, that live on the web and need to be resolvable. A DOI resolves to a particular article, which is selfcontained - it is the object. In contrast, an antibody does not exist on the web but is an independent entity that has data about it scattered across various articles. There is no single digital record that is the antibody; there are documents and data about the entity. We note that in our community we also do not use DOIs to identify people, but rather an ORCID, which serves the same purpose as the RRID.

A case could be made for using DOIs to identify particular software tools and databases, as they are digital objects. As discussed in the next section, our preference is that DOIs be used to identify the particular instance used, e.g., the version of data or software and any supporting workflows, and that the RRID be used to identify the entity or project referenced. Thus, the RRID would be used to identify the Protein Databank, and a PDB identifier or a DOI used to reference the specific data from the PDB. However, we believe that if the RRID system is adopted, appropriate identifier systems should be set by each community. The RRID syntax is meant to be simple and generic and could, in theory, work with any identifier system.
Why coarse granularity?

RRIDs are meant to identify research resources at a fairly high level of granularity. At some of the planning meetings, there was a push for more granular information, like lot and batch numbers, for antibodies. We recognize that this level of granularity is likely an important factor in determining how a given reagent performs ${ }^{10}$. However, in the analysis by Vasilevsky et al. (2013) and in our experience in resource identification using text-mining, the biggest problem was not that authors were not supplying lot numbers but that they were not even supplying catalog numbers. Given that the catalog numbers themselves do not serve as stable identifiers, because antibodies are bought and sold and redistributed by many vendors, we elected to tackle the problem of identifying the root antibody first, i.e., a particular clone for a monoclonal antibody or a type of polyclonal antibody produced by particular protocol. To illustrate the problem, consider the study by Slotta ${ }^{10}$ that provided an analysis of the performance of antibodies to NF-K $\beta$ p65, as a follow up to a similar study by Herkenham ${ }^{4}$. Both studies performed specificity tests on a variety of antibodies and, as is common, did not produce concordant results on all of them. Slotta had been the original producer of an antibody now commonly known as MAB3026 (AB_2178887) and provided its provenance: "It was transferred to Boehringer-Mannheim as Clone $12 \mathrm{H} 11$, resold to Roche and finally bought by Chemicon, and it is now sold as MAB3026." They then speculate that a mutation may have crept in at some point that altered the specificity of the antibody. However, it may simply be that as the antibody was tested under additional conditions, problems were revealed that had not been apparent during more limited applications. The RRID for this antibody binds these different representations together so that all references to this antibody can be tracked. However, authors are encouraged in the citation format to include details about the particular instance of this antibody, namely, the vendor from which the antibody was purchased and the catalog, batch, and lot numbers. However, we did not want to overload the ID system to require assignment of these different lot numbers different RRIDs and maintain the mappings. We also felt that this would grossly decrease compliance.

Similarly, for software and databases, we elected to identify just the root entity and not a granular citation of a particular software version or database. Our main goal in the case of software tools and databases was to track broad patterns of utilization of these resources (e.g., how many times NeuroMorpho.org was used, as per mandate of the NIF, and not particular versions). More complete practices for citing software and data sets are emerging from recent efforts like the Joint Declaration of Data Citation principles (https:// www.force11.org/datacitation), the W3C HCLS dataset description (http://tiny.cc/hcls-datadesc), the software discovery index (http:// softwarediscoveryindex.org/), and many others. In these cases, groups are exploring more complete reporting standards for the individual instances (versions, workflows, virtual machines) that can be used to replicate the findings.

\section{What are the next steps?}

The RII is an example of a grass-roots type of organization that took advantage of existing investments by the NIH to solve a problem without extensive new infrastructure. The RII is continuing to run and has expanded beyond the initial participants. We believe that 
the growth of the initiative indicates that it fills a need not currently met by our existing practices and infrastructure.

Should RRIDs be adopted broadly across all of biomedicine? We would argue yes, the RRID syntax should become the standard for reporting on usage of research resources. We have shown that the requirements for this type of broad adoption are: the availability of a comprehensive and authoritative registry for the appropriate entities; a centralized portal or services that aggregate these registries into a single search call, and the willingness of a community including journals and publishers to support this style of reporting. More sophisticated services can be built to improve and automate authoring and editorial oversight, but these are not required. The solution is therefore accessible both to large commercial publishers, and smaller community- or society-based journals.

If RRIDs were to be broadly adopted tomorrow, what are the outstanding issues regarding implementation and scalability? The first issue is one of scope. The current RII focused on three types of research resources which were broadly used and a known source of variability within experiments. Should all research resources be similarly identified, i.e., every chemical, salt, instrument? We think such an approach would be clumsy and difficult to implement. We can imagine a future where all reagents and tools are bar coded and scanned as they are used in a study. However, as long as humans are responsible for supplying identifiers, we think that the effort should focus on certain types of known problematic entities for which better metadata and ability to query across papers is required. Given the recent problems associated with certain cell lines, for example, these are obvious candidates ${ }^{6}$. The advantage of the current system is that it allows communities who have taken the steps to aggregate and organize resources that are of use to them to agree to include the RRID syntax and single entry point.

The second issue is governance. We deliberately designed a decentralized system that gives control of issuing identifiers to multiple authorities. Such a model requires some governance, in the form of willingness of the authorities to maintain the integrity of any identifiers and links and implementation of a policy regarding entities that are no longer available. We would also need some governance to ensure that multiple, uncoordinated authorities are not issuing IDs for the same research resource and that the IDs assigned to each entity are unique. The latter constraint is handled by the centralized aggregation service currently provided by SciCrunch, however it may be handled by Identifiers.org or other services in the future. Further, the RRID project promotes consistent citation of research resources at a first level identifiability. We believe that more granular reporting standards can and should work hand in hand with the RRIDs and could be coordinated with the authoritative communities, for example, versioned software releases in GitHub.

We believe that the RRID project lays an important foundation for creating a type of "universal product code" (UPC) to help alert the scientific community when issues are raised about key research resources. Reagents and tools are not perfect and problems can arise, as the resources themselves can have issues as they are tested across various paradigms and systems. Even when a resource initially performed well, due to spontaneous mutations in biological resources and interactions between particular software tools and platforms, problems can arise over time. For example, two recent papers have published extensive tests showing that common antibodies for $\mathrm{NF}-\mathrm{Kb}$ show non-specificity under some circumstances ${ }^{7,10}$. Many of these antibodies are extensively used in the literature, but readers of a particular article have no way of knowing that concerns have been raised. We have similar examples with software tools ${ }^{3}$, data sets $^{1,5}$ and genetically modified animals ${ }^{2}$. We have an infrastructure in place, CrossMark, to alert readers of a particular article that an addendum or erratum has been posted. The RRID system can serve as the basis for a similar system for research resources.

\section{Author contributions}

$\mathrm{AB}, \mathrm{PRH}, \mathrm{DNK}, \mathrm{NW}$, EZS NV and members of the Resource Identification Initiative (RII) contributed to data gathering. $\mathrm{AB}$ and MB were responsible for portal creation. PRH and MEM acted as advocates for the RII. AB, MEM were responsible for repository management for Scicrunch and the Antibody Registry. JSG was the Scicrunch portal architect. PRH implemented use of RRIDs at the Journal of Comparative Neurology. MEM implemented use of RRIDs for Brain and Behaviour. MP implemented use of RRIDs at F1000Research. ST implemented use of RRIDs at the Journal of Comparative Neurology. DNK implemented the use of RRIDs at NeuroInformatics. SH implemented use of RRIDs at Frontiers and brought together stakeholders at preliminary meetings. NW scripted the model organism data. EZS championed the use of RRIDs at Elsevier, developed the App and created Figure 4. NV and $\mathrm{AB}$ were responsible for data analysis. $\mathrm{AB}, \mathrm{MB}, \mathrm{JSG}, \mathrm{MAH}, \mathrm{PRH}$, MEM and NV prepared the manuscript. Members of the RINL RII wrote blogs and participated in brainstorming meetings for more than a year. All authors have seen and agreed to the final content of the manuscript.

\section{Competing interests}

The authors declared no competing interests.

\section{Grant information}

This work was supported by: an NIF grant to Martone PI (HHSN271200577531C/PHS HHS/United States); a NIDDK grant to Martone PI (1U24DK097771-01); and a grant from Monarch to Haendel PI (5R24OD011883).

I confirm that the funders had no role in study design, data collection and analysis, decision to publish, or preparation of the manuscript.

\section{Acknowledgements}

We would like to thank John Maunsell for his effort in getting this effort going and helpful comments on the paper, as well as the many other editors who wrote letters to their authors and the staff at various publishers and many other community projects that wrote blogs and press releases about this initiative. We would also like to thank NIH and INCF for providing the venues and vision for this pilot project, specifically Dr. Jonathan Pollock, who drove the workshop and saw early the need to bring this particular group together around a central goal of better identification of research resources. We would also like to thank ZFIN, who very early on recognized the need for an RRID system and provided inspiration for this project. 


\section{Supplementary files}

Supplementary data for 'The Resource Identification Initiative: A cultural shift in publishing', including participating papers (RRIDs and papers.csv), Curated RRIDs for antibodies, organisms and software (NV curation Antibodies.csv, NV curation Organisms.csv and NV curation Software.csv), Primary and Secondary antibodies given RRIDs (Primary vs Secondary Abs.csv) and an analysis of the first 100 RRIDs used in published papers (first 100 analysis.csv).

Click here to access the data.

\section{References}

1. Button KS, loannidis JP, Mokrysz C, et al:: Power failure: why small sample size undermines the reliability of neuroscience. Nat Rev Neurosci. 2013; 14(5): $365-76$

PubMed Abstract | Publisher Full Text

2. Cone $\mathrm{AC}, \mathrm{Ambrosi} \mathrm{C}$, Scemes $\mathrm{E}$, et al:: A comparative antibody analysis of pannexin1 expression in four rat brain regions reveals varying subcellular pannexin1 expression in four rat brain regio

PubMed Abstract | Publisher Full Text | Free Full Text

3. Gronenschild EH, Habets $\mathrm{P}$, Jacobs $\mathrm{HI}$, et al:: The effects of FreeSurfer version, workstation type, and Macintosh operating system version on anatomical volume and cortical thickness measurements. PLoS One. 2012; 7(6): e38234.

PubMed Abstract | Publisher Full Text | Free Full Text

4. Herkenham M, Rathore $\mathrm{P}$, Brown $\mathrm{P}$, et al:: Cautionary notes on the use of NF-KB p65 and p50 antibodies for CNS studies. J Neuroinflammation. 2011; 8: 141.

PubMed Abstract | Publisher Full Text | Free Full Text

5. Hupé JM: Statistical inferences under the Null hypothesis: common mistakes and pitfalls in neuroimaging studies. Front Neurosci. 2015; 9: 18. PubMed Abstract | Publisher Full Text | Free Full Text

6. International Cell Line Authentication Committee (ICLAC): Cell line crosscontamination: WSU-CLL is a known derivative of REH and is unsuitable as a model for chronic lymphocytic leukaemia. Leuk Res. 2014; 38(8): 999-1001.

PubMed Abstract | Publisher Full Text

7. Listwak SJ, Rathore $\mathrm{P}$, Herkenham M: Minimal NF-кB activity in neurons.
Neuroscience. 2013: 250: 282-99.

PubMed Abstract | Publisher Full Text | Free Full Text

8. MacLaren DA, Markovic T, Daniels D, et al:: Enhanced consumption of salient solutions following pedunculopontine tegmental lesions. Neuroscience. 2015 284: 381-99.

PubMed Abstract | Publisher Full Text | Free Full Text

9. McIntyre NJ, Fluck R, Mclntyre C, et al:: Treatment needs and diagnosis awareness in primary care patients with chronic kidney disease. $\mathrm{Br} J \mathrm{Gen}$ Pract. 2012; 62(597): e227-32.

PubMed Abstract | Publisher Full Text | Free Full Text

10. Slotta C, Müller J, Tran L, et al:: An investigation of the specificity of research antibodies against NF-кB-subunit p65. J Histochem Cytochem. 2014; 62(2): 157-61.

PubMed Abstract | Publisher Full Text | Free Full Text

11. Vasilevsky NA, Brush $\mathrm{MH}$, Paddock $\mathrm{H}$, et al.: On the reproducibility of science: unique identification of research resources in the biomedical literature. PeerJ. 2013; 1: e148.

PubMed Abstract | Publisher Full Text | Free Full Text

12. Gardner $\mathrm{D}$, Akil H, Ascoli GA, et al.: The neuroscience information framework: a data and knowledge environment for neuroscience. Neuroinformatics. 2008; 6(3): 149-60.

PubMed Abstract | Publisher Full Text | Free Full Text

13. Marenco LN, Wang R, Bandrowski AE, et al:: Extending the NIF DISCO framework to automate complex workflow: coordinating the harvest and integration of data from diverse neuroscience information resources. Front Neuroinform. 2014; 8: 58 . PubMed Abstract | Publisher Full Text | Free Full Text 


\section{Open Peer Review}

\section{Current Peer Review Status:}

\section{Version 1}

Reviewer Report 18 June 2015

https://doi.org/10.5256/f1000research.7039.r8811

(C) 2015 Gunn W. This is an open access peer review report distributed under the terms of the Creative Commons Attribution License, which permits unrestricted use, distribution, and reproduction in any medium, provided the original work is properly cited.

\section{William Gunn}

Mendeley and the Reproducibility Initiative, Mountain View, CA, USA

The aim of this study was to determine the feasibility and utility of asking authors to include RRIDs when submitting a manuscript. The process for making these determinations was well-considered and the experience and professional relationships of the authors helped greatly in enabling the study to reach the number of journals and submissions that it reached. The analysis of the data was described well and carried out well, making it possible to replicate the experiment, in theory. As much of this study did depend on professional relationships, it's not clear if it could be replicated in practice, but given the nature of the study, this is expected. The authors are to be commended in particular for supplying the raw data supporting the analysis in a usable form as supplementary data, This improved my understanding of the work.

With regard to the 4 outcome measures (Compliance, Accuracy, Identifiability, and Utility) the scoring method is clear and well-described, but I felt like there were some important aspects of the data that were not addressed by the authors. With respect to compliance, it could be said that receiving 312 papers with 572 identifiers shows willingness to contribute, but the data in Table 2 suggest that reaching this level of compliance required a very hands-on approach by the editors. This suggests that compliance may be actually fairly hard to get. It's encouraging that 200 new antibody entries were created by authors, but it would have been good to see more data on the prevalence of other resources.

With regard to the accuracy goal, a user study of a group of authors going through the workflow would have a useful addition to the study to help determine where the trouble spots for authors arise. Are they in the lookup on the SciCrunch portal or, as suggested for the case of MGI, at the registry itself?

The identifiability pre-pilot results are consistent with the results we reached in our analysis of the Reproducibility Project: Cancer Biology set of papers. The authors assert that authors are able to perform the task accurately for software tools, but it is worth noting that software tools had low identifiability before and improved the least. Some discussion about why that is the case would be useful. 
Regarding utility, evidence is presented that RRIDs are highly useful because an RRID can be entered into Google Scholar and a link to a paper mentioning that RRID can be retrieved. This is perhaps the weakest part of the study, because the utility criteria were that a query could be constructed to show all the publications in which the resource were used could be found. They report data that only 174 identifiers could be found in Google Scholar, but it's not clear how this data was obtained, not exactly what the numbers refer to. The exact query used for each search index should be supplied \& the results themselves should have been stored. Given the lack of detail around these points, it's hard to judge if it's actually possible to conduct these queries in a systematic fashion. The discussion about the failure of researchers to include RRIDs in a consistent form shows the current method to get RRIDs inserted in the literature is not sufficient for widespread use, and the level of support required by the SciCrunch site maintainers (100 queries for 10000 searches) also suggests that this approach would not scale. Given the lack of an API for Google Scholar, the approach used is understandable, and it's noted that the RRID Resolver page at SciCrunch does link to a PubMed query for papers mentioning the resource, but there is a text mining API available for ScienceDirect papers which would have been a better choice for assessing presence in ScienceDirect. Overall, the study would have been improved by the addition of a data scientist \& must be judged to have failed on the utility criteria as established. This doesn't mean RRIDs are shown not to be useful; on the contrary, it's clear that RRIDs do allow a researcher to find the actual resource used, so in that respect they're quite useful indeed and the resolver page is very helpful. There's a case for broader utility to be made, as well, with the example of the "Antibody data for this article" article enhancement on ScienceDirect.

It would also have been useful to see a more extended discussion of the sustainability of this project. For example, are journals to take this on as a means to add value, and if so, who pays for the maintenance of the SciCrunch resolver?

Overall, the authors have presented good quality data on the feasibility and usability of asking authors to contribute RRIDs to publications and the publishing community should consider this study when considering ways to enrich the literature for text and data mining purposes.

The takeaways for me are:

Just asking authors via the information for authors pages or via one-off emails does not work.

Letting authors enter RRIDs manually detracts from the utility of the identifiers. There's a big opportunity for machine learning approaches here to identify and suggest RRIDs at submission.

Non-open access articles inhibit research by preventing the full-text indexing of their articles by search indexes.

Competing Interests: I am co-founder of the Reproducibility Initiative, so I'm favorably inclined towards anything that looks to improve reproducibility. I also work for Mendeley, a service for researchers which is engaged in text and data mining of literature. Mendeley is owned by Elsevier, which developed the 'Antibody data from this article" widget shown as an example in this paper.

I confirm that I have read this submission and believe that I have an appropriate level of 


\section{expertise to confirm that it is of an acceptable scientific standard.}

Reviewer Report 05 June 2015

https://doi.org/10.5256/f1000research.7039.r8814

(C) 2015 Vita R. This is an open access peer review report distributed under the terms of the Creative Commons Attribution License, which permits unrestricted use, distribution, and reproduction in any medium, provided the original work is properly cited.

\section{Randi Vita}

Division of Vaccine Discovery, La Jolla Institute for Allergy and Immunology, La Jolla, CA, USA

This manuscript is overall well written with an appropriate title. The abstract provides an adequate summary and the design and explanation are sound. I believe the conclusions are justified and the data provided is adequate to support them. The subject is valuable to many researchers and provides an important service to researchers going forward. I have a few minor grammatical issues to report:

1. The sentence "The pilot project has focused on a limited number of resources - antibodies, software tools/databases, and model organisms" should be "The pilot project focused on a limited number of resources..."

2. in "accession number, as Genbank does for gene" should use "GenBank"

3. For sentence "Over the minimum 3-month window, each partner journal would request that authors supply RRIDs in a standard format as a citation to indicate the use of any of these three types of research resources."

In this context, what the "three types of research resources" are is unclear.

4. The sentence "As these papers start to appear in PubMed Central where there is full text search, it should be possible to find papers for RRIDs through the National Library of Medicine." is a little confusing, maybe "As these papers start to appear in PubMed Central, where full text search is possible, papers utilizing RRIDs will be identifiable through the National Library of Medicine." is better? Yes, being overly nit picky, but I had to read it twice to get it.

5. Verb tense should be reviewed throughout the manuscript to ensure consistency.

6. Uses of dashes such as "...from vendors - both solicited and unsolicited - and at.." occurs throughout the manuscript. This seems nonstandard?

7. In Figure 3, I could not see the pre-pilot bar. This could be due to something with my browser, but worth checking out.

8. In the Discussion, the sentence "The pilot RRID project has been highly successful in demonstrating the utility of a system to aid in identification of these three research 
resources in the literature" should reiterate what three research resources it is referring to.

9. Review the number of uses of the word "however" and how close to each other they occur. For example, "However, authors are encouraged in the citation format to include details about the particular instance of this antibody, namely, the vendor from which the antibody was purchased and the catalog, batch, and lot numbers. However, we..."

And lastly, the link referred to here "A list of the participating journals is available on the Force11 website (https://www.force11.org/RII/SignUp)." did not provide a list of the multiple immunology journals in the Elsevier family that are participating. The page just shows the Elsevier logo.

Competing Interests: No competing interests were disclosed.

I confirm that I have read this submission and believe that I have an appropriate level of expertise to confirm that it is of an acceptable scientific standard.

The benefits of publishing with F1000Research:

- Your article is published within days, with no editorial bias

- You can publish traditional articles, null/negative results, case reports, data notes and more

- The peer review process is transparent and collaborative

- Your article is indexed in PubMed after passing peer review

- Dedicated customer support at every stage

For pre-submission enquiries, contact research@f1000.com 